\title{
Association of jumping mechanography-derived indices of muscle function with tibial cortical bone geometry
}

\author{
Authors \\ Charlotte Verroken \\ Hans-Georg Zmierczak \\ Stefan Goemaere \\ Jean-Marc Kaufman \\ Bruno Lapauw
}

\section{Affiliation}

Unit for Osteoporosis and Metabolic Bone Diseases, Department of Endocrinology

Ghent University Hospital

De Pintelaan 185, 9K12IE

9000 Ghent

Belgium

\section{Corresponding author}

Charlotte Verroken, MD

Department of Endocrinology, Ghent University Hospital

De Pintelaan 185, 9K12IE

B - 9000 Gent

Belgium

Charlotte.Verroken@UGent.be

Tel: +3293321966

Fax: +3293323897

\section{Acknowledgements}

The authors thank K. Toye and K. Mertens for their technical assistance, Dr. E. Deschepper for statistical consulting and the volunteers for participation in this study. The study was supported by a grant from the Fund for Scientific Research - Flanders (FWO-Vlaanderen, grant \#G.0867.11).

The final publication is available at Springer via http://dx.doi.org/10.1007/s00223-015-0094-2 


\begin{abstract}
Jumping mechanography has been developed to estimate maximum voluntary muscle forces. This study assessed associations of jumping mechanography-derived force and power measurements with tibial cortical bone geometry, compared to other estimates of muscle mass, size and function. Healthy men ( $n=181 ; 25-45$ years) were recruited in a cross-sectional, population-based sibling-pair study. Muscle parameters include isokinetic peak torque of the quadriceps, DXA-derived leg lean mass, mechanography-derived peak jump force and power and pQCT-derived mid-tibial (66\%) muscle cross-sectional area (CSA). Mid-tibial cortical bone parameters were assessed by pQCT. In age, height and weight-adjusted analyses, jump force and power correlated positively with cortical bone area, cortical thickness and polar strength strain index (SSIp) ( $\beta=0.23-0.34, p \leq 0.001$ for force; $\beta=0.25-0.30, p \leq 0.007$ for power), and inversely with endosteal circumference adjusted for periosteal circumference $\left(E C_{P C}\right)(\beta=-0.16, p<0.001$ for force; $\beta=-0.13, p=0.007$ for power). Force but not power correlated with cortical over total bone area ratio $(\beta=0.25, p=0.002)$. Whereas leg lean mass correlated with all cortical parameters except cortical over total bone area ratio ( $B=0.25$ $0.62, p \leq 0.004)$, muscle CSA only correlated with cortical bone area, periosteal circumference and SSIp ( $ß=0.21-0.26, p \leq 0.001)$, and quadriceps torque showed no significant correlations with the bone parameters. Multivariate models indicated that leg lean mass was independently associated with overall bone size and strength reflected by periosteal and endosteal circumference and SSIp ( $B=0.32$ $0.55, p \leq 0.004)$, whereas jump force was independently associated with cortical bone size reflected by $E C_{P C}$, cortical thickness and cortical over total bone area ratio ( $\left.\beta=0.13-0.28 ; p \leq 0.002\right)$. These data indicate that jumping mechanography provides relevant information about the relationship of muscle with bone geometry.
\end{abstract}

KEY WORDS: jumping mechanography; peak force; mechanical loading; bone geometry; bone strength; male; $\mathrm{pQCT}$ 


\section{INTRODUCTION}

The strength of a bone depends on its material properties on the one hand and on the amount of bone tissue and its distribution in the bone organ (architectural properties or bone geometry) on the other hand [1]. In healthy subjects the material properties vary little with age and are only in part affected by external factors, whereas the amount of bone tissue and its geometry largely depend on lifelong modeling processes activated by mechanical load-induced deformations (strains) [2, 3] besides factors such as hormone exposure, inflammatory status, co-morbidities and drug use. In everyday life, the largest strains result from regional muscle contractions [4-7]. When investigating functional muscle-bone relationships, it is therefore important to evaluate the maximum forces that can be produced by the muscles acting on the bone in question [8]. However, since maximum intrinsic muscle forces cannot be directly measured under in vivo conditions, estimates of muscle mass or size (e.g. muscle volume, cross-sectional area, or lean mass) as well as estimates of isokinetic muscle function (e.g. peak torque) have been frequently used as surrogate markers. Nonetheless, their reflection of maximum voluntary muscle force or their practical use are often limited and as such, the use of these assessments in the evaluation of muscle-bone relationships is rather questionable. More recently, jumping mechanography has been proposed as a more accurate method to estimate the maximum intrinsic forces that can be voluntarily generated in the leg musculature, by measuring ground reaction forces [8]. This technique is highly reproducible and can be safely used in populations covering a wide range of age and body size [9-12].

According to the mechanostat theory [2], strains caused by muscle forces influence bone geometry and therefore, mechanography-derived muscle force estimates might correlate better with bone geometry than regional muscle mass, volume, cross-sectional area, or peak torque. Although previous studies have indeed shown positive associations of peak jump force and/or power with bone size and strength [13-18], studies comparing jumping mechanography to other estimates of muscle size and function are scarce and inconclusive $[13-15,17]$. Moreover, some of these studies used non-commercial ground reaction force plates [14-16] or suboptimal jump procedures [13-16], failed to account for body mass $[14,15,17]$, or investigated specific populations $[13,18]$. The present study therefore aims to examine the associations of mechanography-derived peak jump force and power, as compared to other commonly used muscle mass, size and function estimates, with tibial cortical bone geometry in young, healthy men. We hypothesized that (1) peak jump force would correlate better with bone geometry than peak jump power, as strains are caused by force and not power; and that (2) peak jump force would correlate better with bone geometry and strength than other muscle size and function estimates only indirectly associated with strain. 


\section{SUBJECTS AND METHODS}

\subsection{SUBJECTS}

This study is part of a population-based study (SIBLOS) designed to investigate determinants of peak bone mass in men, focusing on general lifestyle, sex hormone status, body composition, and genetic background. The detailed study design has been described previously [19]. Briefly, 1114 healthy men aged 25-45 years, who had a brother within the same age range also willing to participate, were recruited from the population registries of the semi-rural to urban communities around Ghent, Belgium, between March, 2002 and July, 2010. After implementation of the exclusion criteria (including illnesses or medication use affecting body composition, sex hormone status or bone metabolism), 1001 men were included in the study cohort. The study protocol was approved by the ethical committee of the Ghent University Hospital and written informed consent was obtained from all participants. As jumping mechanography assessments were implemented in the study protocol in 2008, the current study is based on data of the 181 men included from January, 2008 onwards. Physical activity was scored using the questionnaire as proposed by Baecke et al. [20]. All assessments were performed by a well-trained and dedicated study team.

\subsection{ANTHROPOMETRY AND ESTIMATES OF MUSCLE SIZE AND FUNCTION}

Body weight was measured to the nearest $0.1 \mathrm{~kg}$ in light indoor clothing without shoes. Standing height was measured to the nearest $0.1 \mathrm{~cm}$ using a wall-mounted Harpenden stadiometer (Holtain Ltd., Crymuch, UK). Body composition was measured using dual-energy X-ray absorptiometry (DXA) with a Hologic QDR-4500A device (software version 11.2.1; Hologic, Bedford, MA, USA), with leg lean mass $(\mathrm{kg})$ defined as the lean mass from both lower limbs including thigh musculature. The coefficient of variation (CV) for whole-body calibration phantoms was less than $1 \%$ as calculated from weekly measurements; CVs for outcome variables have not been calculated. Muscle cross-sectional area $\left(\mathrm{CSA}, \mathrm{cm}^{2}\right)$ was assessed at the dominant tibial shaft (66\% of bone length from distal end) using a peripheral quantitative computed tomography (pQCT) device (XCT-2000; Stratec Medizintechnik, Pforzheim, Germany) as described below, with $\mathrm{CV}$ for the calibration phantom $<1 \%$ as calculated from daily measurements. Isokinetic peak torque of the quadriceps muscle $(\mathrm{Nm})$ was assessed during knee extension movements of the dominant limb at a preset constant angular velocity of $60 \% / \mathrm{sec}$, using an isokinetic dynamometer (Biodex, New York, NY, USA). Three trial efforts and five maximal efforts with no rest were allowed to produce five overlying curves, and peak torque was derived from the effort with the highest value. Jumping mechanography was performed using a Leonardo Mechanography Ground Reaction Force Platform (software version 4.2; Novotec Medical GmbH, Pforzheim, Germany). As recommended by the manufacturer, peak jump force $(\mathrm{kN})$ was assessed during multiple 1-legged hopping (m1LH) on the dominant limb, whereas peak jump power (kW) was 
assessed during single 2-legged jumps (s2 LJ). For the $\mathrm{m} 1 \mathrm{LH}$, participants were instructed to bounce on the ball of the foot with the knee almost straight and the ankle joint as stiff as possible, aiming to achieve a maximum ground reaction force. The jump with the highest vertical ground reaction force was selected for analysis. The $\mathrm{s} 2 \mathrm{LJ}$ was performed as a countermovement jump for which individuals were asked to jump as high as possible, producing a maximal elevation of the center of mass. This jump was repeated 3 times and the recording with the highest jump height was analyzed. Both jump procedures were performed with freely moving arms. The CV for peak jump power was $5.08 \%$ as calculated from 3 repeated jumps for all participants, CV for peak jump force has not been calculated.

\subsection{BONE GEOMETRY}

Geometric bone parameters and estimates of bone strength were determined at the dominant lower leg (tibial shaft, $66 \%$ of bone length from distal end) using pQCT. Single tomographic slices of $2.0 \mathrm{~mm}$ thickness were taken at a voxel size of $0.8 \mathrm{~mm}$ and a scan speed of $20 \mathrm{~mm} / \mathrm{sec}$. Imaging and the calculation of numerical values were performed using the manufacturer's software package (version 5.4). The following parameters were assessed: total bone area $\left(\mathrm{mm}^{2}\right)$, cortical bone area $\left(\mathrm{mm}^{2}\right)$, periosteal circumference $(\mathrm{mm})$, endosteal circumference $(\mathrm{mm})$, cortical thickness $(\mathrm{mm})$, and polar strength-strain index $\left(\mathrm{SSIp}, \mathrm{mm}^{3}\right)$. CSA of the tibia was determined after detecting the outer bone contour at a threshold of $280 \mathrm{mg} / \mathrm{cm}^{3}$. Periosteal and endosteal circumference and cortical thickness were estimated using a circular ring model, with cortical thickness as the difference between the outer and inner radius. The cortical over total bone area ratio (\%) was calculated as cortical bone area divided by total bone area. Whereas we considered the total bone area and periosteal circumference as indicators of overall bone size, the cortical over total bone area ratio and cortical thickness rather reflect the amount of cortical bone. Muscle CSA was estimated using a threshold below water equivalent linear attenuation set at $0.22 / \mathrm{cm}$. This threshold eliminated skin and fat mass with lower linear attenuation in the cross-sectional slice. From the remaining area, bone area was subtracted, showing the muscle at its maximum CSA. The CV for the calibration phantom was < $1 \%$ as calculated from daily measurements; CVs for outcome variables have not been calculated.

\subsection{STATISTICS}

Descriptives are expressed as mean \pm standard deviation (SD). Visual inspection of histograms and quantile-quantile (QQ) plots and Shapiro-Wilk tests were performed to assess normality. Linear mixed-effects modeling with a variance components residual correlation structure for random effects was used to evaluate cross-sectional relationships and differences between the participants who did and those who did not perform jumping mechanography, taking the interdependence of 
measurements within families into account. Continuous variables were standardized in order to obtain standardized regression coefficients. Parameters of fixed effects were estimated using maximum likelihood estimation and reported as standardized regression coefficients (ß) with their respective 95\% confidence intervals $(\mathrm{Cl})$. Unless stated otherwise, analyses are adjusted for age, height and weight. Analyses including endosteal circumference were additionally adjusted for periosteal circumference to provide an estimate of endosteal expansion independently of bone size $\left(E C_{P C}\right)$ [18]. To determine the independent associations of the different muscle parameters with each of the bone parameters, multivariate models were constructed with one of the bone parameters as the dependent and all muscle parameters showing a significant correlation with the considered bone parameter as the predictor variables, with additional adjustment for age, height and weight ('Enter' method). No multicollinearity issues were detected based on the correlation coefficients between the predictors, $95 \% \mathrm{Cl}^{\prime}$, and since linear mixed models do not provide collinearity diagnostics, collinearity diagnostics derived from linear regression models performed in a random sample including one participant from every family. Considering the large number of statistical tests, the significance level for associations was arbitrarily set at $p<0.01$; all $p$ values were two-tailed. $\mathrm{R}^{2}$ statistics were derived from models considering one of the bone parameters as the dependent and one of the muscle parameters in addition to age, height and weight as the predictor variables and were calculated as $R^{2}=1-\exp \left(-2\left(\log L_{m}-\log L_{0}\right) / n\right)$, where $\log L_{m}$ denotes the log-likelihood of the model of interest, $\log \mathrm{L}_{0}$ denotes the log-likelihood of an intercept-only model, and $\mathrm{n}$ denotes the number of observations [21]. The relative quality of these models was assessed by comparing Akaike Information Criteria (AIC), with the lowest AIC indicating the best fitting model. All analyses were performed using SPSS version 22.0 (IBM Corp., Armonk, NY, USA). 


\section{RESULTS}

\subsection{DESCRIPTION OF THE STUDY POPULATION}

The study population comprised 181 participants who performed jumping mechanography, including 73 brother pairs, 2 triplets and 29 singles. Their general characteristics as well as estimates of muscle size and function and parameters reflecting bone geometry are summarized in Table 1. All participants completed the s2LJ, 178 participants completed the $\mathrm{m} 1 \mathrm{LH}$. Leg lean mass, muscle CSA and quadriceps peak torque were available for 178,181 and 174 participants, respectively. The majority of the participants (63.5\%) had a normal BMI, $11.0 \%$ were overweight (BMI $25-30 \mathrm{~kg} / \mathrm{m}^{2}$ ), and $6.7 \%$ were obese $\left(\mathrm{BMI} \geq 30 \mathrm{~kg} / \mathrm{m}^{2}\right)$. As compared to the participants who did not perform jumping mechanography, participants included in this study had somewhat higher periosteal $(97 \pm 6$ $\mathrm{mm}$ vs. $95 \pm 6 \mathrm{~mm}, \mathrm{p}<0.001)$ and endosteal ( $69 \pm 7 \mathrm{~mm}$ vs. $67 \pm 7 \mathrm{~mm}, \mathrm{p}<0.001)$ circumferences. No other relevant differences were detected.

Table 2 displays correlations between the different muscle parameters. The strongest associations were seen between leg lean mass and peak jump power and between leg lean mass and muscle CSA, whereas the weakest correlation was observed between muscle CSA at the tibial shaft and quadriceps torque. Overall, the strength of the correlations was moderate with a maximal correlation coefficient of 0.69 between leg lean mass and peak jump power.

\subsection{ASSOCIATIONS OF JUMPING MECHANOGRAPHY WITH BONE GEOMETRY}

In unadjusted analyses, both peak jump force and power were positively associated with cortical bone area ( $\beta=0.42$ for force; $\beta=0.42$ for power, both $p<0.001$ ), cortical thickness ( $\beta=0.31, p<0.001$ and $\beta=0.23, p=0.002$ ), periosteal circumference $(\beta=0.28$ and $\beta=0.39$, both $p<0.001)$ and $S S I p(\beta=0.35$ and $\beta=0.44$, both $p<0.001)$, and negatively with $E C_{P C}(\beta=-0.16$ and $\beta=-0.13$, both $p<0.001$; Figure 1$)$. In addition, power but not force displayed a positive correlation with endosteal circumference ( $\beta=0.24$, $p=0.001$ ), whereas force but not power tended to be associated with the cortical over total bone area ratio $(B=0.16, p=0.022)$. As shown in Table 3 , the associations of force and power with cortical bone area, cortical thickness, SSIp and $\mathrm{EC}_{\mathrm{PC}}$ as well as the association of force with the cortical over total bone area ratio remained significant after adjustment for age, height and weight, whereas the correlations of force and power with periosteal circumference and the correlation of power with endosteal circumference weakened and lost significance. For cortical bone area, cortical thickness, $\mathrm{EC}_{\mathrm{PC}}$ and SSIp, models including peak jump force fitted better than models including peak jump power, with $\mathrm{R}^{2}$ values $40.5 \%$ vs. $35.4 \%, 23.6 \%$ vs. $15.3 \%, 26.2 \%$ vs. $20.3 \%$, and $48.9 \%$ vs. $46.7 \%$, respectively. Moreover, when force and power were both included in the regression models, only force was independently associated with these bone parameters $(B=0.29$ for cortical area, $\beta=0.30$ for 
cortical thickness, $\beta=-0.14$ for $E C_{P C}$ and $\beta=0.12$ for $S S I p$, all $p<0.001$ ). Additional adjustment for physical activity level did not alter the results (data not shown).

\subsection{ASSOCIATIONS OF OTHER MUSCLE PARAMETERS WITH BONE GEOMETRY}

In unadjusted analyses, muscle CSA, quadriceps torque and leg lean mass correlated positively with cortical bone area ( $\beta=0.37, p<0.001$ for muscle $C S A ; \beta=0.25, p=0.001$ for quadriceps torque; $\beta=0.56$, $p<0.001$ for leg lean mass), periosteal $(\beta=0.34 ; \beta=0.36$ and $\beta=0.64$, all $p<0.001)$ and endosteal circumference $(\beta=0.21, p=0.002 ; \beta=0.27, p<0.001$ and $\beta=0.45, p<0.001)$, and $\operatorname{SSI}(\beta=0.38 ; \beta=0.33$ and $\beta=0.64$, all $p<0.001$ ). In addition, muscle CSA and leg lean mass correlated positively with cortical thickness $(B=0.18, p=0.008$ and $\beta=0.23, p=0.001)$ and negatively with $E C_{P C}(\beta=-0.10, p=0.002$ and $\beta=-$ $0.19, p<0.001)$. No associations were found between any of the muscle parameters and the cortical over total bone area ratio. After adjustment for body weight, height and age, the associations of muscle CSA with cortical bone area, periosteal circumference, $\mathrm{SSIp}$ and $\mathrm{EC}_{\mathrm{PC}}$ weakened but remained significant (borderline for $\mathrm{EC}_{\mathrm{PC}}$ ), whereas the associations with endosteal circumference and cortical thickness lost significance (Table 3). The associations of leg lean mass with cortical bone area, cortical thickness and $E_{P C}$ became somewhat stronger, whereas those with periosteal and endosteal circumference and SSIp weakened but remained significant. None of the correlations between quadriceps torque and bone geometry parameters remained significant.

\subsection{INDEPENDENT ASSOCIATIONS OF THE DIFFERENT MUSCLE PARAMETERS WITH BONE GEOMETRY}

Table 4 displays the results of the multivariate analyses assessing the independent relations of the muscle parameters with bone geometry. Whereas leg lean mass correlated independently with periosteal and endosteal circumference and SSIp, peak jump force was independently associated with the cortical over total bone area ratio, cortical thickness and $E C_{P C}$. Both force and leg lean mass were independently associated with cortical bone area. 


\section{DISCUSSION}

The present study showed that mechanography-derived peak jump force and, albeit not independently from force, power correlate positively with parameters reflecting cortical bone geometry and strength at the tibial shaft. This correlation seems driven by an association with decreased endosteal expansion rather than increased periosteal apposition, as reflected by the inverse correlations with $\mathrm{EC}_{\mathrm{PC}}$. Regarding other muscle mass, size and function estimates, leg lean mass strongly correlates with parameters reflecting overall as well as cortical bone size and bone strength, while the associations of muscle CSA and especially quadriceps torque with bone geometry seem less consistent. Multivariate modeling revealed that whereas leg lean mass is independently associated with overall bone size and strength, peak jump force specifically and independently correlates to cortical bone size. These data thus suggest that as compared to other commonly used muscle size and function estimates, jumping mechanography provides a better estimation of the maximum voluntary muscle forces affecting cortical bone parameters.

Confirming our hypothesis and broadly consistent with previous studies [13-18], peak jump force was a stronger predictor of bone geometry than peak jump power. In contrast, some authors observed similar or stronger correlations of bone size and strength with power than with force [13, 15], although their use of single two-legged jumps to assess jump force may have resulted in an underestimation of peak forces [8]. To our knowledge, only one other study investigated the associations of peak jump force and power with endosteal expansion, reporting an inverse relationship of endosteal expansion with peak jump power but not force [18]. However, they studied a more heterogeneous population including a large number of high bone mass cases, who differ from the general population in their cortical phenotype [22]. Moreover, we found higher mean values for peak jump power and force, explained by our younger and only male study population. More evidence supporting the hypothesis of a particular association between muscle force and endosteal expansion results from studies on physical activity and bone geometry, demonstrating that vigorous but not moderate or light habitual physical activity is associated with reduced endosteal expansion [23] and that sports requiring very high muscle forces (e.g. hurdling and triple jump), as compared to lower intensity sports, are associated with larger differences in cortical area and thickness than in total cross-sectional bone area $[24,25]$. As such, locally exerted strains, as reflected by peak jump force, particularly appear to affect cortical bone geometry.

Leg lean mass and muscle CSA were associated with parameters reflecting cortical as well as overall bone geometry, which is largely in line with previous studies [3,13-15, 17, 19, 26-34]. However, the associations of muscle CSA with bone geometry were not independent of jump force or leg lean 
mass, and quadriceps torque showed no correlations with bone geometry at all. Stronger associations of bone strength and size with peak jump force than with muscle CSA have also been described by Anliker et al. [17], but possibly due to the use of less indicative jump procedures, could not or only in part be confirmed by other authors [13-15]. Differences in explanatory capacity of jump force versus muscle CSA and quadriceps torque with respect to cortical bone geometry might be explained by several factors. First, muscle CSA and quadriceps torque are poor surrogate markers of muscle force as they correlate only moderately to peak jump force and power. On the one hand, although isokinetic dynamometry theoretically represents a good method for the evaluation of leg muscle function, as maximum muscle forces are generated during eccentric contractions $[8,17]$, it is a rather impractical technique yielding a large variability in peak torque output due to methodological difficulties $[8,35]$. On the other hand, the use of muscle size measurements such as muscle CSA as a surrogate of maximum intrinsic muscle force is limited, because the mechanical potential of a muscle depends not only on morphology but also on muscle fibre type and muscle composition [36]. As known from biopsies, spectroscopy and MRI studies, fatty infiltration of muscles can already be noticed in young, healthy subjects, confounding the relationship between the size of a muscle and the forces that can be generated by its fibres [37, 38]. Moreover, peak jump force is determined by factors such as Achilles tendon elasticity and joint size. Since joint size is determined at the end of puberty and afterwards cannot be enhanced, peak joint forces must be controlled in order not to exceed those that the joints had adapted to at that time, to avoid rupturing of the tendons. Increases in muscle mass or size may therefore not lead to increases in maximum muscle force in healthy, non-deconditioned adults $[8,39]$. Second, the peak torque measurements in this study were derived from the quadriceps muscle whereas the bone geometry was assessed at the tibia, and the absence of any association may therefore not be entirely unexpected. Third, besides being a (poor) marker of muscle force, muscle CSA is also a surrogate of lean mass, which might affect bone geometry in a different way than muscle force. Indeed, in contrast to muscle CSA and quadriceps torque and in line with the results of Binkley et al. [13], leg lean mass was strongly associated with peak jump force and power as well as with bone geometry and strength. Moreover, and as opposed to our hypothesis, it was the only muscle parameter independently associated with periosteal circumference and thus overall bone size. Although strong correlations of (appendicular) lean mass with bone geometry have been previously described, the mechanisms underlying these associations are incompletely understood $[13,32,33,40]$. In general, DXA-derived estimates of leg lean mass reflect a combination of both potential local muscle activity and the overall individual's health status, body composition and physical activity level. We speculate that total leg lean mass better represents local muscle activity than a single cross-sectional measurement of the muscle, even if the latter is assessed in the same area as the bone geometry. In addition, the muscle secretes a 
variety of cytokines and growth factors collectively referred to as myokines, which might modulate bone metabolism through endocrine and other signaling pathways [41]. Furthermore, certain regulatory pathways and associated factors, such as androgens, affect both skeletal muscle mass and (directly or indirectly) bone size, and evidence exists that myogenic progenitor cells may play a role in bone formation [42].

In a very simplified model, it can be assumed that under physiological conditions, factors such as hormones and myokines acting in an endocrine manner and/or through specific tissue-tissue transport mechanisms mainly influence overall bone size, whereas the detailed shaping of bones involved in motion is mainly controlled locally through strain-sensing by osteocytes and consecutive effector mechanisms (in turn probably modulated by systemic factors). Further, while overall bone size, as reflected by periosteal circumference, in adult life mainly reflects influences of the past (e.g. during growth), cortical area, cortical thickness and endosteal circumference are much more subject to change. Possibly, leg lean mass reflects the systemic factors and past influences on the tibia better than the other examined muscle parameters, whereas peak jump force may be the better indicator of strain-mediated adaptive changes. This might explain the pronounced independent associations of leg lean mass with overall bone size as opposed to the associations of jump force with cortical bone size.

Our findings imply that jumping mechanography and leg lean mass measurements should be considered advantageous methods in future studies investigating functional muscle bonerelationships, given their distinct associations with cortical and overall bone geometry. Clearly, further research is needed to elucidate to what extent interventions addressing peak voluntary muscle force influence cortical bone geometry and fracture risk, as this would have an impact on rehabilitation programs or other interventions for patients with suspected bone deficits. Given the fact that fracture risk seems to be mainly predicted by cortical rather than overall bone size [43-48], we speculate that increasing peak voluntary muscle force would benefit bone health and fracture risk more than increasing (leg) lean mass.

A major strength of this study is the well-defined population-based sample of healthy men. Since all study subjects were between 25 and 45 years of age, we assume that they had already reached peak bone mass but were not yet subjected to major degenerative alterations of the skeletal or the muscular system at the time of the study. Moreover, we used several methods to estimate muscle size and function including jumping mechanography, which enabled us to compare this more recent technique with other commonly used measurements. In contrast to previous studies, peak jump force, muscle CSA and bone parameters were assessed at the same (dominant) limb. Our study also 
has limitations. Obviously, the cross-sectional design does not allow to draw conclusions about causality. Although our sample size was relatively small compared to some studies focusing on traditional muscle function measurements and bone parameters, only two studies using jumping mechanography investigated larger, but also more heterogeneous populations [16, 17]. Furthermore, the peak torque measurements were derived during knee extension and therefore mainly reflect muscles at the upper leg, whereas the bone parameters were measured at the mid tibia. Torque measurements derived from ankle plantarflexion might have been more informative and more strongly correlated to tibial bone strength and geometry; however, such measurements were not available in our study population. The relation of bone strength with peak jump force as well as with ankle plantarflexion torque has been assessed in one study, observing stronger associations of plantarflexion torque with compressive bending strength but not overall bone strength [14]. Nonetheless, as mentioned earlier, the use of two-legged instead of one-legged hopping to assess jump force might have lead to an underestimation of peak forces in this study.

In conclusion, our results indicate that out of several commonly used muscle mass, size and function estimates, leg lean mass independently correlates with overall bone size and strength at the tibial shaft, whereas the forces exerted on the bone, as represented by mechanography-derived peak jump force, specifically correlate with cortical bone size. As compared to other muscle parameters, jumping mechanography thus provides relevant additional information about the relationship of muscle with cortical bone geometry. 


\section{CONTRIBUTORS}

Jean-Marc Kaufman, Hans-Georg Zmierczak, Stefan Goemaere designed the study. Hans-Georg Zmierczak, Stefan Goemaere, Bruno Lapauw and Jean-Marc Kaufman collected the data. Charlotte Verroken was responsible for statistical analysis of the data and wrote the first draft of the paper. She is guarantor. All authors revised the paper critically for intellectual content and approved the final version. All authors agree to be accountable for the work and ensure that any questions relating to the accuracy and integrity of the paper are investigated and properly resolved.

\section{ACKNOWLEDGEMENTS}

The authors thank K. Toye and K. Mertens for their technical assistance, Dr. E. Deschepper for statistical consulting and the volunteers for participation in this study. The study was supported by a grant from the Fund for Scientific Research - Flanders (FWO-Vlaanderen, grant \#G.0867.11).

\section{COMPLIANCE WITH ETHICAL STANDARDS}

Conflict of interest Charlotte Verroken, Hans-Georg Zmierczak, Stefan Goemaere, Jean-Marc Kaufman and Bruno Lapauw declare that they have no conflict of interest.

Human rights and informed consent The study was conducted in accordance with the 1964 Helsinki declaration and its later amendments and the study protocol was approved by the ethical committee of the Ghent University Hospital. Written informed consent was obtained from all individual participants included in the study. 


\section{REFERENCES}

1. Özkaya N, Nordin M, Goldsheyder D, Leger D (2001) Fundamentals of Biomechanics: Equilibrium, Motion, and Deformation, 3rd edn. Springer, New York

2. Frost HM (1997) Why do marathon runners have less bone than weight lifters? A vitalbiomechanical view and explanation. Bone 20:183-9

3. Rittweger J, Beller G, Ehrig J, Jung C, Koch U, Ramolla J, Schmidt F, Newitt D, Majumdar S, Schiessl H, Felsenberg D (2000) Bone-muscle strength indices for the human lower leg. Bone 27:319-26

4. Ferretti JL, Cointry GR, Capozza RF, Frost HM (2003) Bone mass, bone strength, muscle-bone interactions, osteopenias and osteoporoses. Mech Ageing Dev 124:269-279. doi:

10.1016/S0047-6374(02)00194-X

5. Frost HM (2003) Bone's mechanostat: a 2003 update. Anat Rec A Discov Mol Cell Evol Biol 275:1081-101. doi: 10.1002/ar.a.10119

6. Fricke O, Schoenau E (2007) The "Functional Muscle-Bone Unit": probing the relevance of mechanical signals for bone development in children and adolescents. Growth Horm IGF Res 17:1-9. doi: 10.1016/j.ghir.2006.10.004

7. Robling AG (2009) Is bone's response to mechanical signals dominated by muscle forces? Med Sci Sport Exerc 41:2044-2049. doi: 10.1249/MSS.0b013e3181a8c702.Is

8. Anliker E, Toigo M (2012) Functional assessment of the muscle-bone unit in the lower leg. J Musculoskelet Neuronal Interact 12:46-55

9. Rittweger J, Schiessl H, Felsenberg D, Runge M (2004) Reproducibility of the jumping mechanography as a test of mechanical power output in physically competent adult and elderly subjects. J Am Geriatr Soc 52:128-131

10. Veilleux LN, Rauch F (2010) Reproducibility of jumping mechanography in healthy children and adults. J Musculoskelet Neuronal Interact 10(4):256-66

11. Buehring B, Krueger D, Fidler E, Gangnon R, Heiderscheit B, Binkley N (2014) Reproducibility of jumping mechanography and traditional measures of physical and muscle function in older adults. Osteoporos Int 26:819-825. doi: 10.1007/s00198-014-2983-z

12. Buehring B, Krueger D, Binkley N (2010) Jumping mechanography: a potential tool for sarcopenia evaluation in older individuals. J Clin Densitom 13:283-291. doi: 10.1016/j.jocd.2010.04.002

13. Binkley TL, Specker BL (2008) Muscle-bone relationships in the lower leg of healthy prepubertal females and males. J Musculoskelet Neuronal Interact 8:239-43

14. Rantalainen $T$, Heinonen A, Komi P, Linnamo V (2008) Neuromuscular performance and bone structural characteristics in young healthy men and women. Eur J Appl Physiol 102(2):215-22. doi: 10.1007/s00421-007-0575-8 
15. Rantalainen $T$, Sievänen $H$, Linnamo V, Hoffrén $M$, Ishikawa $M$, Kyröläinen $H$, Avela J, Selänne $\mathrm{H}$, Komi PV, Heinonen A (2009) Bone rigidity to neuromuscular performance ratio in young and elderly men. Bone 45:956-63. doi: 10.1016/j.bone.2009.07.014

16. Rantalainen T, Nikander R, Heinonen A, Multanen J, Häkkinen A, Jämsä T, Kiviranta I, Linnamo V, Komi PV, Sievänen $H$ (2010) Neuromuscular performance and body mass as indices of bone loading in premenopausal and postmenopausal women. Bone 46:964-9. doi:

10.1016/j.bone.2010.01.002

17. Anliker E, Rawer R, Boutellier U, Toigo M (2011)Maximum ground reaction force in relation to tibial bone mass in children and adults. Med Sci Sports Exerc 43:2102-9. doi:

10.1249/MSS.0b013e31821c4661

18. Hardcastle SA, Gregson CL, Rittweger J, Crabtree N, Ward K, Tobias JH (2014) Jump power and force have distinct associations with cortical bone parameters: findings from a population enriched by individuals with high bone mass. J Clin Endocrinol Metab 99:266-75. doi: 10.1210/jc.2013-2837

19. Lapauw BM, Taes Y, Bogaert V, Vanbillemont G, Goemaere S, Zmierczak HG, De Bacquer D, Kaufman JM (2009) Serum estradiol is associated with volumetric BMD and modulates the impact of physical activity on bone size at the age of peak bone mass: a study in healthy male siblings. J Bone Miner Res 24:1075-85. doi: 10.1359/JBMR.081260

20. Baecke JA, Burema J, Frijters JE (1982) A short questionnaire for the measurement of habitual physical activity in epidemiological studies. Am J Clin Nutr 36:936-42

21. Nakagawa S, Schielzeth $H$ (2013) A general and simple method for obtaining $R^{2}$ from generalized linear mixed-effects models. Methods Ecol Evol 4:133-142. doi: 10.1111/j.2041210x.2012.00261.x

22. Gregson CL, Sayers A, Lazar V, Steel S, Dennison EM, Cooper C, Smith GD, Rittweger J, Tobias $\mathrm{JH}$ (2013) The high bone mass phenotype is characterised by a combined cortical and trabecular bone phenotype: findings from a pQCT case-control study. Bone 52:380-8. doi: 10.1016/j.bone.2012.10.021

23. Sayers A, Mattocks C, Deere K, Ness A, Riddoch C, Tobias JH (2011) Habitual levels of vigorous, but not moderate or light, physical activity is positively related to cortical bone mass in adolescents. J Clin Endocrinol Metab 96:E793-802. doi: 10.1210/jc.2010-2550

24. Nikander R, Kannus P, Rantalainen T, Uusi-Rasi K, Heinonen A, Sievänen H (2010) Crosssectional geometry of weight-bearing tibia in female athletes subjected to different exercise loadings. Osteoporos Int 21:1687-1694. doi: 10.1007/s00198-009-1101-0

25. Heinonen A, Sievänen H, Kyröläinen H, Perttunen J, Kannus P (2001) Mineral mass, size, and estimated mechanical strength of triple jumpers' lower limb. Bone 29:279-285

26. Ashe MC, Liu-Ambrose TY, Cooper DM, Khan KM, McKay HA (2008) Muscle power is related to tibial bone strength in older women. Osteoporos Int 19:1725-32. doi: 10.1007/s00198-0080655-6 
27. Högler W, Blimkie CJ, Cowell CT, Inglis D, Rauch F, Kemp AF, Wiebe P, Duncan SC, FarpourLambert N, Woodhead HJ (2008) Sex-specific developmental changes in muscle size and bone geometry at the femoral shaft. Bone 42:982-9. doi: 10.1016/j.bone.2008.01.008

28. Bailey CA, Brooke-Wavell K (2010) Association of body composition and muscle function with hip geometry and BMD in premenopausal women. Ann Hum Biol 37:524-535. doi: 10.3109/03014460903443207

29. Cousins JM, Petit MA, Paudel ML, Taylor BC, Hughes JM, Cauley JA, Zmuda JM, Cawthon PM, Ensrud KE for the Osteoporotic Fractures in Men (MrOS) Study Group (2010) Muscle power and physical activity are associated with bone strength in older men: The osteoporotic fractures in men study. Bone 47:205-11. doi: 10.1016/j.bone.2010.05.003

30. Wetzsteon RJ, Zemel BS, Shults J, Howard KM, Kibe LW, Leonard MB (2011) Mechanical loads and cortical bone geometry in healthy children and young adults. Bone 48:1103-8. doi: 10.1016/j.bone.2011.01.005

31. Lebrasseur NK, Achenbach SJ, Melton LJ, Amin S, Khosla S (2012) Skeletal muscle mass is associated with bone geometry and microstructure and serum insulin-like growth factor binding protein-2 levels in adult women and men. J Bone Miner Res 27:2159-69. doi: 10.1002/jbmr.1666

32. Baker JF, Davis M, Alexander R, Zemel BS, Mostoufi-Moab S, Shults J, Sulik M, Schiferl DJ, Leonard MB (2013) Associations between body composition and bone density and structure in men and women across the adult age spectrum. Bone 53:34-41. doi:

10.1016/j.bone.2012.11.035

33. Farr JN, Amin S, LeBrasseur NK, Atkinson EJ, Achenbach SJ, McCready LK, Joseph Melton L 3rd, Khosla S (2014) Body composition during childhood and adolescence: relations to bone strength and microstructure. J Clin Endocrinol Metab 99:4641-8. doi: 10.1210/jc.2014-1113

34. Liu PY, Hornbuckle LM, Ilich JZ, Kim JS, Panton L (2014) Body composition and muscular strength as predictors of bone mineral density in African American women with metabolic syndrome. Ethn Dis 24:356-362

35. Olmo J, Jato S, Benito J, Martín I, Dvir Z (2009) Identification of feigned ankle plantar and dorsiflexors weakness in normal subjects. J Electromyogr Kinesiol 19:774-81. doi: 10.1016/j.jelekin.2008.02.001

36. Noble JJ, Charles-Edwards GD, Keevil SF, Lewis AP, Gough M, Shortland AP (2014) Intramuscular fat in ambulant young adults with bilateral spastic cerebral palsy. BMC Musculoskelet Disord 15:236. doi: 10.1186/1471-2474-15-236

37. Manini TM, Clark BC, Nalls MA, Goodpaster BH, Ploutz-snyder LL, Harris TB (2007) Reduced physical activity increases intermuscular adipose tissue in heatlhy young adults. Am J Clin Nutr 85:377-84

38. Kim HK, Serai S, Merrow AC, Wang L, Horn PS, Laor T (2014) Objective measurement of minimal fat in normal skeletal muscles of healthy children using $T 2$ relaxation time mapping (T2 maps) and MR spectroscopy. Pediatr Radiol 44:149-57. doi: 10.1007/s00247-013-2791-1 
39. Rittweger J (2008) Ten years muscle-bone hypothesis: what have we learned so far? - Almost a Festschrift. J Musculoskelet Neuronal Interact 8:174-178

40. Taes YE, Lapauw B, Vanbillemont G, Bogaert V, De Bacquer D, Zmierczak H, Goemaere S, Kaufman JM (2009) Fat mass is negatively associated with cortical bone size in young healthy male siblings. J Clin Endocrinol Metab 94:2325-31. doi: 10.1210/jc.2008-2501

41. Hamrick MW (2011) A role for myokines in muscle-bone interactions. Exerc Sport Sci Rev 39:43-47. doi: 10.1097/JES.0b013e318201f601

42. Henrotin $Y(2011)$ Muscle: a source of progenitor cells for bone fracture healing. BMC Med 9:136. doi: 10.1186/1741-7015-9-136

43. Vilayphiou N, Boutroy S, Szulc P, Van Rietbergen B, Munoz F, Delmas PD, Chapurlat R (2011) Finite element analysis performed on radius and tibia HR-pQCT images and fragility fractures at all sites in men. J Bone Miner Res 26:965-73. doi: 10.1002/jbmr.297

44. Joseph Melton L 3rd, Lawrence Riggs B, Keaveny TM, Achenbach SJ, Hoffmann PF, Camp JJ, Rouleau PA, Bouxsein ML, Amin S, Atkinson EJ, Robb RA, Khosla S (2007) Structural Determinants of Vertebral Fracture Risk. J Bone Miner Res 22:1885-92. doi: 10.1359/jbmr.070728

45. Stein EM, Liu XS, Nickolas TL, Cohen A, Thomas V, McMahon DJ, Zhang C, Yin PT, Cosman F, Nieves J, Guo XE, Shane E (2011) Abnormal microarchitecture and stiffness in postmenopausal women with ankle fractures. J Clin Endocrinol Metab 96:2041-48. doi: 10.1210/jc.2011-0309

46. Stein EM, Kepley A, Walker M, Nickolas TL, Nishiyama K, Zhou B, Liu XS, McMahon DJ, Zhang C, Boutroy S, Cosman F, Nieves J, Guo XE, Shane E (2014) Skeletal structure in postmenopausal women with osteopenia and fractures is characterized by abnormal trabecular plates and cortical thinning. J Bone Miner Res 29:1101-09. doi: 10.1002/jbmr.2144

47. Dennison EM, Jameson KA, Edwards MH, Denison HJ, Aihie Sayer A, Cooper C (2014) Peripheral quantitative computed tomography measures are associated with adult fracture risk: the Hertfordshire Cohort Study. Bone 64:13-17. doi: 10.1016/j.bone.2014.03.040

48. Sheu Y, Zmuda JM, Boudreau RM, Petit MA, Ensrud KE, Bauer DC, Gordin CL, Orwoll ES, Cauley JA for the Osteoporotic Fractures in Men (MrOS) Research Group (2011) Bone strength measured by peripheral quantitative computed tomography and the risk of nonvertebral fractures: the osteoporotic fractures in men (MrOS) study. J Bone Miner Res 26:63-71. doi: 10.1002/jbmr.172 


\section{FIGURES}

Fig. 1 Scatter plots with regression lines and 95\% confidence intervals representing unadjusted associations between mechanography-derived peak jump force and bone geometry
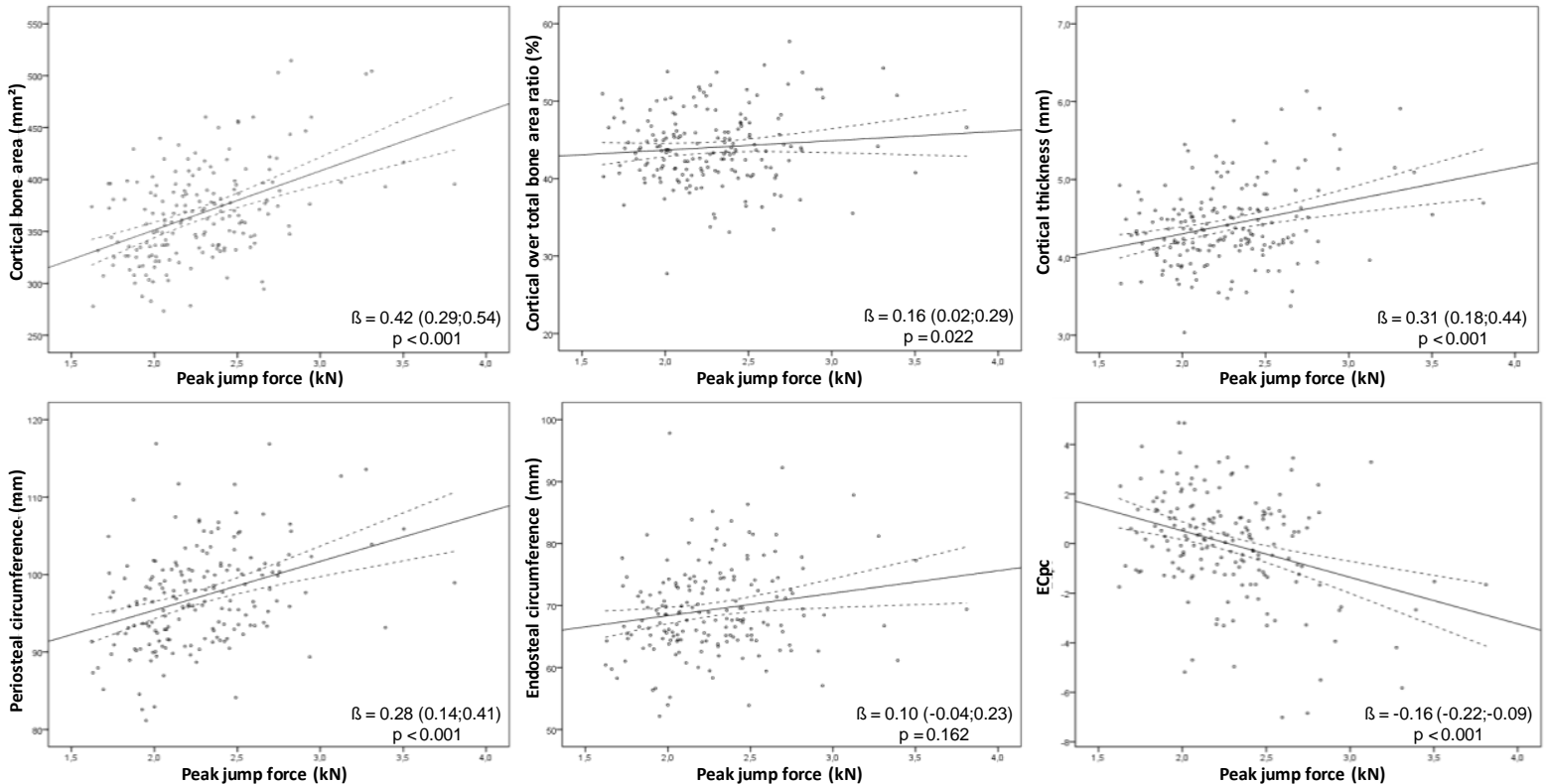


\section{TABLES}

Table 1 General characteristics, estimates of muscle size and function, and parameters reflecting bone geometry and strength $(n=181)$

\begin{tabular}{lc}
\hline & Mean \pm SD \\
\hline General characteristics & $34.1 \pm 5.4$ \\
Age (yr) & $79.4 \pm 11.1$ \\
Weight (kg) & $180.3 \pm 6.9$ \\
Height (cm) & $24.4 \pm 3.2$ \\
BMI (kg/m ${ }^{2}$ ) & $2.64 \pm 0.40$ \\
Mean activity index (score) & \\
Muscle parameters & $0.52 \pm 0.09$ \\
Maximum jump height (m) & $4.0 \pm 0.7$ \\
Peak jump power (kW) & $51 \pm 8$ \\
Relative peak jump power (W/kg) & $2.3 \pm 0.4$ \\
Peak jump force (kN) & $2.9 \pm 0.4$ \\
Relative peak jump force (times Fg) & $197 \pm 42$ \\
Quadriceps peak torque (Nm) & $83 \pm 12$ \\
Muscle CSA at tibial shaft (cm ${ }^{2}$ ) & $19.9 \pm 2.7$ \\
Leg lean mass (kg) & \\
Bone parameters & $367 \pm 45$ \\
Cortical bone area (mm ${ }^{2}$ ) & $44 \pm 5$ \\
Cortical over total bone area ratio (\%) & $97 \pm 6$ \\
Periosteal circumference (mm) & $69 \pm 7$ \\
Endosteal circumference (mm) & $4.4 \pm 0.5$ \\
Cortical thickness (mm) & \\
SSl (mm ${ }^{3}$ ) & \\
\hline
\end{tabular}

Fg: gravitational force, CSA: cross-sectional area, $\mathrm{SSI}_{\mathrm{p}}$ : polar strength strain index. 
Table 2 Correlations between muscle size and function estimates

\begin{tabular}{|c|c|c|c|c|c|c|c|c|}
\hline & \multicolumn{2}{|l|}{ pPs2L } & \multicolumn{2}{|l|}{ pFm1LH } & \multicolumn{2}{|c|}{ Leg lean mass } & \multicolumn{2}{|c|}{ Quadriceps torque } \\
\hline & ß $(95 \% \mathrm{Cl})$ & $p$ & B $(95 \% \mathrm{Cl})$ & $p$ & B $(95 \% \mathrm{Cl})$ & $p$ & ß $(95 \% \mathrm{Cl})$ & $\mathrm{p}$ \\
\hline Muscle CSA & $0.39(0.26 ; 0.51)$ & $<0.001$ & $0.42(0.30 ; 0.55)$ & $<0.001$ & $0.61(0.50 ; 0.71)$ & $<0.001$ & $0.23(0.09 ; 0.36)$ & 0.001 \\
\hline Quadriceps torque & $0.38(0.24 ; 0.51)$ & $<0.001$ & $0.35(0.21 ; 0.50)$ & $<0.001$ & $0.43(0.29 ; 0.57)$ & $<0.001$ & & \\
\hline Leg lean mass & $0.69(0.59 ; 0.79)$ & $<0.001$ & $0.59(0.47 ; 0.70)$ & $<0.001$ & & & & \\
\hline pFm1LH & $0.46(0.34 ; 0.57)$ & $<0.001$ & & & & & & \\
\hline
\end{tabular}

Analyses are unadjusted. Dependent and predictor variables were standardized in order to obtain standardized regression coefficients (B). pPs2L: peak jump power (single 2-legged jump), pFm1LH: peak jump force (multiple 1-legged hopping), CSA: cross sectional area. 
Table 3 Associations of muscle parameters with bone geometry

\begin{tabular}{|c|c|c|c|c|c|c|c|c|c|c|}
\hline & \multicolumn{2}{|l|}{ pPs2L } & \multicolumn{2}{|l|}{ pFm1LH } & \multicolumn{2}{|c|}{ Muscle CSA } & \multicolumn{2}{|c|}{ Quadriceps torque } & \multicolumn{2}{|c|}{ Leg lean mass } \\
\hline & B $(95 \% \mathrm{Cl})$ & $\mathrm{p}$ & B $(95 \% \mathrm{Cl})$ & $\mathrm{p}$ & B $(95 \% \mathrm{Cl})$ & $\mathrm{p}$ & B $(95 \% \mathrm{Cl})$ & $\mathrm{p}$ & B $(95 \% \mathrm{Cl})$ & $\mathrm{p}$ \\
\hline Cortical bone area & $0.28(0.12 ; 0.44)$ & 0.001 & $0.33(0.19 ; 0.47)$ & $<0.001$ & $0.26(0.13 ; 0.40)$ & $<0.001$ & $0.08(-0.06 ; 0.23)$ & ns & $0.62(0.41 ; 0.82)$ & $<0.001$ \\
\hline Cortical over total bone area ratio & $0.16(-0.02 ; 0.35)$ & ns & $0.25(0.09 ; 0.41)$ & 0.002 & $0.02(-0.14 ; 0.18)$ & ns & $-0.07(-0.23 ; 0.09)$ & ns & $0.10(-0.15 ; 0.35)$ & ns \\
\hline Periosteal circumference & $0.13(-0.02 ; 0.27)$ & ns & $0.11(-0.02 ; 0.25)$ & ns & $0.21(0.09 ; 0.33)$ & 0.001 & $0.15(0.02 ; 0.28)$ & ns & $0.56(0.38 ; 0.74)$ & $<0.001$ \\
\hline Endosteal circumference & $-0.002(-0.17 ; 0.16)$ & ns & $-0.06(-0.21 ; 0.09)$ & ns & $0.12(-0.02 ; 0.26)$ & ns & $0.10(-0.04 ; 0.24)$ & ns & $0.32(0.11 ; 0.54)$ & 0.004 \\
\hline $\mathrm{EC}_{\mathrm{PC}}$ & $-0.13(-0.21 ;-0.04)$ & 0.003 & $-0.16(-0.23 ;-0.09)$ & $<0.001$ & $-0.09(-0.16 ;-0.02)$ & ns & $-0.01(-0.08 ; 0.06)$ & ns & $-0.25(-0.36 ;-0.13)$ & $<0.001$ \\
\hline Cortical thickness & $0.25(0.07 ; 0.44)$ & 0.007 & $0.34(0.18 ; 0.49)$ & $<0.001$ & $0.15(-0.01 ; 0.31)$ & ns & $0.01(-0.15 ; 0.17)$ & ns & $0.37(0.12 ; 0.61)$ & 0.003 \\
\hline SSIp & $0.30(0.10 ; 0.51)$ & 0.003 & $0.23(0.10 ; 0.36)$ & 0.001 & $0.26(0.14 ; 0.38)$ & $<0.001$ & $0.13(-0.0002 ; 0.26)$ & ns & $0.61(0.44 ; 0.79)$ & $<0.001$ \\
\hline
\end{tabular}

Analyses are adjusted for age, height and weight. Dependent and predictor variables were standardized in order to obtain standardized regression coefficients ( $(3)$. pPs2 $\sqcup$ : peak jump power (single 2-legged jump), pFm1LH: peak jump force (multiple 1-legged hopping), CSA: cross-sectional area, $\mathrm{EC}_{\mathrm{PC}}$ : endosteal circumference additionally adjusted for periosteal circumference, SSIp: polar strength strain index, ns: non-significant. 
Table 4 Independent associations of muscle parameters with bone geometry

\begin{tabular}{|c|c|c|c|c|c|c|c|c|}
\hline & \multicolumn{2}{|l|}{ pPs2L } & \multicolumn{2}{|l|}{ pFm1LH } & \multicolumn{2}{|l|}{ Muscle CSA } & \multicolumn{2}{|c|}{ Leg lean mass } \\
\hline & B $(95 \% \mathrm{Cl})$ & $\mathrm{p}$ & B $(95 \% \mathrm{Cl})$ & $\mathrm{p}$ & B $(95 \% \mathrm{Cl})$ & $\mathrm{p}$ & B $(95 \% \mathrm{Cl})$ & $\mathrm{p}$ \\
\hline Cortical bone area & $0.06(-0.11 ; 0.23)$ & ns & $0.22(0.08 ; 0.36)$ & 0.002 & $0.08(-0.07 ; 0.23)$ & ns & $0.42(0.16 ; 0.68)$ & 0.002 \\
\hline Cortical over total bone area ratio & - & & $0.25(0.09 ; 0.41)$ & 0.002 & - & & - & \\
\hline Periosteal circumference & - & & - & & $0.02(-0.12 ; 0.16)$ & ns & $0.55(0.33 ; 0.77)$ & $<0.001$ \\
\hline Endosteal circumference & - & & - & & - & & $0.32(0.11 ; 0.54)$ & 0.004 \\
\hline $\mathrm{EC}_{\mathrm{PC}}$ & $-0.04(-0.13 ; 0.05)$ & ns & $-0.13(-0.20 ;-0.05)$ & 0.001 & - & & $-0.16(-0.29 ; 0.03)$ & ns \\
\hline Cortical thickness & $0.10(-0.10 ; 0.30)$ & ns & $0.28(0.11 ; 0.44)$ & 0.001 & - & & $0.18(-0.09 ; 0.45)$ & ns \\
\hline SSIp & $0.02(-0.14 ;-0.17)$ & ns & $0.12(-0.01 ; 0.25)$ & ns & $0.08(-0.08 ; 0.19)$ & ns & $0.51(0.27 ; 0.75)$ & $<0.001$ \\
\hline
\end{tabular}

Models include one of the bone parameters as the dependent and the indicated muscle parameters as the independent variables and are additionally adjusted for age, height and weight. Inclusion of the muscle parameters was based on their significant correlation with the dependent variable in age, height and weight adjusted analyses. Dependent and predictor variables were standardized in order to obtain standardized regression coefficients (B). pPs2L: peak jump power (single 2-legged jump), pFm1LH: peak jump force (multiple 1-legged hopping), CSA: cross-sectional area, $\mathrm{EC}_{\mathrm{p}}$ : endosteal circumference additionally adjusted for periosteal circumference, SSIp: polar strength strain index, ns: non-significant. 